\title{
Correction to: Radiological exploration on adjacent segments after total cervical disc replacement with Prodisc- $C$ prosthesis
}

Shuai $\mathrm{Xu}^{\dagger}$, Yan Liang ${ }^{\dagger}$, Fanqi Meng, Kaifeng Wang and Haiying Liu ${ }^{*}$

\author{
Comection to: J Orthop Surg Res (2019) 14:160 \\ https://doi.org/10.1186/s13018-019-1194-x
}

In the original publication of this article [1], the Table 1, 2, 3, 4 and 5 were missing due to a mistake in the copy-editing stage.

Table 1, 2, 3, 4 and 5 are listed below:

The original article has been corrected.

Published online: 08 July 2019

\section{Reference}

1. $\mathrm{Xu}$, et al. Radiological exploration on adjacent segments after total cervical disc replacement with Prodisc-C prosthesis. J Orthop Surg Res. 2019;14:160 https://josr-online.biomedcentral.com/articles/10.1186/s13018-019-1194-x.

*Correspondence: liuhaiying1131@sina.com

tShuai Xu and Yan Liang contributed equally to this work.

Department of Spinal Surgery, Peking University People's Hospital, Peking

University, No. 11 Xizhimen South Street, Xicheng District, Beijing 100044,

People's Republic of China 
Table 1 Demographic characteristics and CDDD information of patients

\begin{tabular}{|c|c|}
\hline Parameters & Statistics \\
\hline No. of patients & 118 \\
\hline \multicolumn{2}{|l|}{ Gender } \\
\hline Male & $65(55.1 \%)$ \\
\hline Female & $53(44.9 \%)$ \\
\hline Age (years) & $46.85 \pm 9.38$ \\
\hline BMI (kg/m2) & $24.62 \pm 3.72$ \\
\hline Follow-up (months) & $135.75 \pm 8.46$ \\
\hline \multicolumn{2}{|l|}{ Diagnosis } \\
\hline Radiculopathy & 39 (33.0\%) \\
\hline Myelopathy & 27 (22.9\%) \\
\hline Radiculopathy \& Myelopathy & $52(44.1 \%)$ \\
\hline \multicolumn{2}{|l|}{ No. of levels } \\
\hline Single level & $90(76.3 \%)$ \\
\hline Double levels & $20(16.9 \%)$ \\
\hline Three levels & $8(6.78 \%)$ \\
\hline \multicolumn{2}{|l|}{ Distribution of operated levels } \\
\hline$C 3 / 4$ & $10(6.5 \%)$ \\
\hline $\mathrm{C} 4 / 5$ & $32(20.8 \%)$ \\
\hline$C 5 / 6$ & $90(58.4 \%)$ \\
\hline $\mathrm{C} 6 / 7$ & $22(14.3 \%)$ \\
\hline \multicolumn{2}{|l|}{ Distribution of adjacent segments } \\
\hline $\mathrm{C} 2 / 3$ & $10(4.2 \%)$ \\
\hline $\mathrm{C} 3 / 4$ & $30(12.7 \%)$ \\
\hline $\mathrm{C} 4 / 5$ & $76(32.2 \%)$ \\
\hline$C 5 / 6$ & $21(8.9 \%)$ \\
\hline $\mathrm{C} 6 / 7$ & 77 (32.6\%) \\
\hline $\mathrm{C} 7 / \mathrm{T} 1$ & $22(9.3 \%)$ \\
\hline
\end{tabular}

Footnote: CDDD Cervical disc degeneration disease, No. Number, BMI Body mass index

Table 2 Radiographic primary outcomes from preoperation to the final FU

\begin{tabular}{|c|c|c|c|c|c|c|c|c|}
\hline & Preoperation & 1-week FU & 6-month FU & 1-year FU & 2-year FU & 5-year FU & 10-year FU & Final FU \\
\hline \multicolumn{9}{|l|}{$\mathrm{ROM}\left({ }^{\circ}\right)$} \\
\hline UAS & $9.10 \pm 5.78$ & $6.43 \pm 4.40^{* *}$ & $7.83 \pm 5.36$ & $7.96 \pm 4.16^{*}$ & $7.72 \pm 4.97$ & $7.60 \pm 4.44$ & $7.59 \pm 3.86$ & $7.38 \pm 3.55^{*}$ \\
\hline LAS & $7.94 \pm 5.95$ & $6.57 \pm 4.98$ & $7.61 \pm 5.32$ & $8.14 \pm 5.76$ & $8.61 \pm 5.61^{*}$ & $8.45 \pm 5.16$ & $7.41 \pm 4.31$ & $7.71 \pm 4.33$ \\
\hline \multicolumn{9}{|l|}{$\operatorname{LOR}\left(^{\circ}\right)$} \\
\hline UAS & $3.28 \pm 5.82$ & $2.28 \pm 5.36$ & $2.80 \pm 5.00$ & $3.64 \pm 4.97$ & $4.75 \pm 7.34$ & $5.84 \pm 5.50$ & $3.75 \pm 3.46$ & \\
\hline LAS & $3.91 \pm 5.32$ & $2.94 \pm 5.21$ & $3.94 \pm 5.66$ & $4.15 \pm 5.15$ & $3.16 \pm 5.64$ & $3.98 \pm 4.03$ & $5.03 \pm 4.95$ & \\
\hline \multicolumn{9}{|c|}{$\mathrm{IDH}(\mathrm{mm})$} \\
\hline UAS & $4.72 \pm 0.66$ & $4.76 \pm 0.71$ & $4.71 \pm 0.74$ & $4.66 \pm 0.71$ & $4.69 \pm 0.72$ & $4.71 \pm 0.70$ & $4.71 \pm 0.72$ & $4.69 \pm 0.72$ \\
\hline LAS & $4.73 \pm 0.95$ & $4.95 \pm 1.10$ & $4.77 \pm 0.96$ & $4.78 \pm 1.01$ & $4.83 \pm 0.90$ & $4.73 \pm 1.06$ & $4.64 \pm 0.95$ & $4.66 \pm 0.96$ \\
\hline
\end{tabular}

Footnote: FU Follow up, ROM Range of motion, UAS Upper adjacent segment, LAS Lower adjacent segment, LOR Lordosis, IDH Intervertebral disc height *: Significance $(\mathrm{P}<0.05)$ in comparison to baseline

**: Significance $(P<0.01)$ in comparison to baseline 
Table 3 Pearson correlation analysis among primary outcomes

\begin{tabular}{|c|c|c|c|c|c|c|c|}
\hline & & UAS-ROM & LAS-ROM & UAS-LOR & LAS-LOR & UAS-IDH & LAS-IDH \\
\hline \multirow[t]{2}{*}{ UAS-ROM } & r & 1 & 0.206 & 0.043 & 0.014 & 0.018 & 0.011 \\
\hline & $P$ Value & & 0.000 & 0.547 & 0.857 & 0.692 & 0.814 \\
\hline \multirow[t]{2}{*}{ LAS-ROM } & $r$ & & 1 & 0.085 & 0.007 & -0.054 & 0.104 \\
\hline & $P$ Value & & & 0.236 & 0.933 & 0.245 & 0.025 \\
\hline \multirow[t]{2}{*}{ UAS-LOR } & r & & & 1 & 0.362 & 0.025 & 0.114 \\
\hline & $P$ Value & & & & 0.000 & 0.727 & 0.113 \\
\hline \multirow[t]{2}{*}{ LAS-LOR } & r & & & & 1 & 0.115 & 0.154 \\
\hline & $P$ Value & & & & & 0.148 & 0.052 \\
\hline \multirow[t]{2}{*}{ UAS-IDH } & r & & & & & 1 & 0.451 \\
\hline & $P$ Value & & & & & & 0.000 \\
\hline
\end{tabular}

Footnote: UAS Upper adjacent segment, ROM Range of motion, LAS Lower adjacent segment, LOR Lordosis, IDH Intervertebral disc height

Table 4 Correlation analysis between primary outcomes and secondary outcomes

\begin{tabular}{lllllllllll}
\hline & & C2-C7 ROM & OP-ROM & C2-C7 LOR & OP-LOR & OP-IDH & Subsidence & Cor-M & Sag-M & HO \\
\hline UAS-ROM & $r$ & 0.573 & 0.465 & 0.128 & -0.043 & -0.075 & -0.027 & -0.041 & 0.095 & -0.068 \\
& $P$ Value & 0.000 & 0.000 & 0.005 & 0.506 & 0.102 & 0.589 & 0.408 & 0.054 & 0.167 \\
LAS-ROM & $r$ & 0.513 & 0.395 & 0.069 & 0.024 & 0.003 & -0.060 & 0.028 & 0.052 & 0.128 \\
& $P$ Value & 0.000 & 0.000 & 0.140 & 0.706 & 0.951 & 0.226 & 0.573 & 0.295 & 0.010 \\
UAS-LOR & $r$ & 0.086 & 0.117 & 0.096 & 0.179 & -0.038 & 0.183 & 0.124 & 0.086 & 0.072 \\
& $P$ Value & 0.237 & 0.108 & 0.188 & 0.014 & 0.603 & 0.033 & 0.146 & 0.313 & 0.404 \\
LAS-LOR & $r$ & 0.052 & 0.160 & 0.057 & 0.085 & 0.027 & 0.061 & 0.051 & -0.001 & -0.029 \\
& $P$ Value & 0.524 & 0.048 & 0.483 & 0.294 & 0.743 & 0.522 & 0.593 & 0.991 & 0.758 \\
UAS-IDH & $r$ & 0.011 & 0.032 & -0.029 & 0.005 & 0.070 & 0.018 & 0.042 & 0.043 & -0.071 \\
& $P$ Value & 0.804 & 0.484 & 0.526 & 0.942 & 0.129 & 0.718 & 0.397 & 0.379 & 0.152 \\
LAS-IDH & $r$ & 0.057 & 0.131 & 0.089 & 0.058 & 0.179 & 0.150 & 0.019 & -0.033 & -0.173 \\
& $P$ Value & 0.214 & 0.004 & 0.053 & 0.373 & 0.000 & 0.002 & 0.696 & 0.500 & 0.000 \\
\hline
\end{tabular}


Table 5 Multiple linear regression analysis of primary outcomes

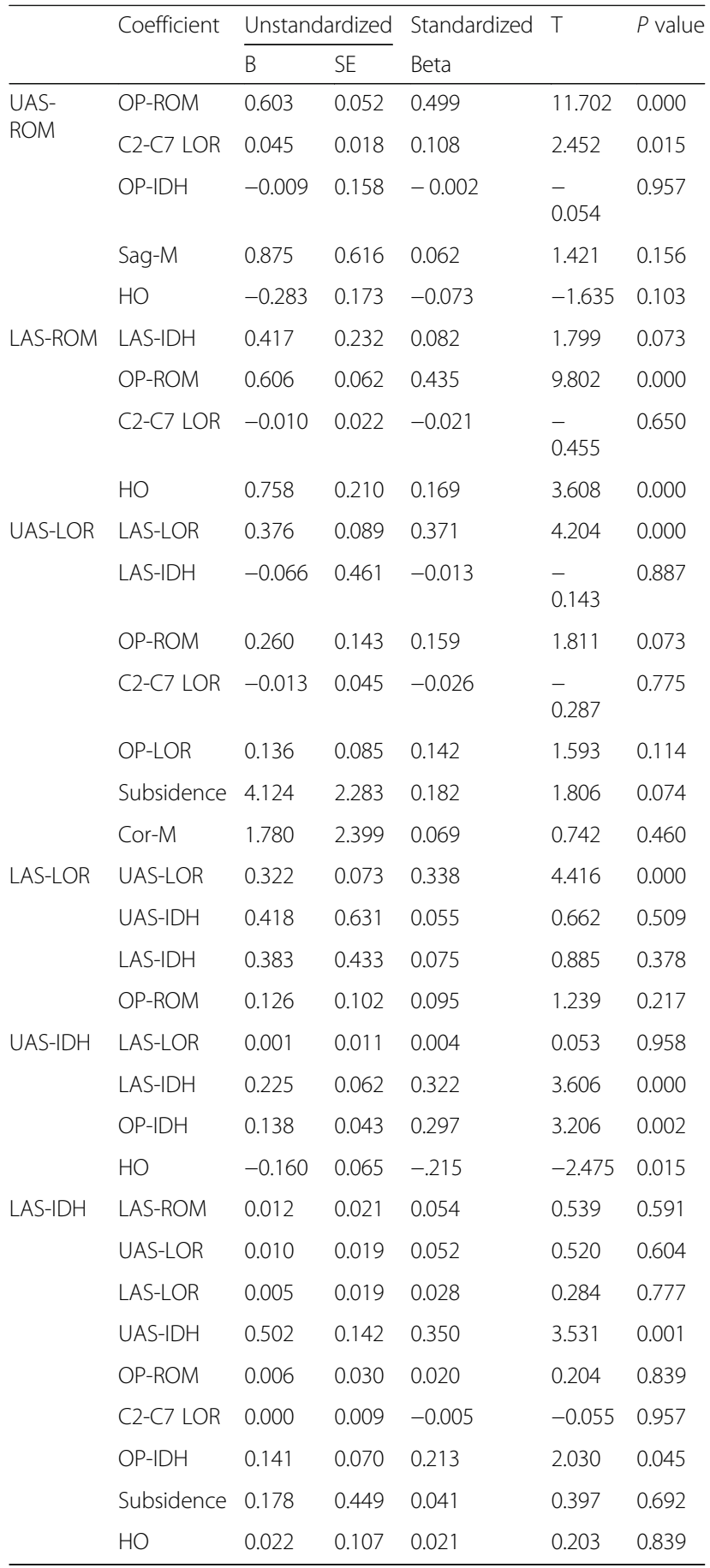

Footnote: SE Standard error, UAS Upper adjacent segment, ROM Range of motion, LAS Lower adjacent segment, LOR Lordosis, IDH Intervertebral disc height, OP Operation segment, Sag-M Sagittal migration, HO Heterotopic ossification, Cor-M Coronal migration 